\title{
Monopropellant droplet decomposition for large activation energies
}

\author{
AMABLE LIÑ ÁN†
}

\begin{abstract}
The quasi-steady decomposition of a monopropellant droplet in a quiescent atmosphere is analyzed, in the limit of large activation energy, for a gas phase decomposition reaction of the Arrhenius type.

An analytical relation is obtained for the pre-exponential rate constant, or the Damköhler number, as a function of the droplet vaporization rate. The curve giving the vaporization rate in terms of the Damköhler number has, for low values of the temperature at infinity, an $\mathrm{S}$ shaped form which exhibits ignition-extinction characteristics.

With increasing values of the droplet vaporization rate above the pure vaporization value we pass from a nearly frozen regime, under which ignition conditions occur due to the large temperature sensitivity of the reaction rate, to a complete decomposition regime, in which practically all the fuel is decomposed in a thin reactive-diffusion zone, separated from the droplet surface by a transport region of lower temperature. For sufficiently large Damköhler numbers, the thin reaction zone is located close to the droplet surface, so that the flame structure becomes planar: a linear relation between the droplet radius and time is obtained in this case. The non planar effects are responsible for the extinction of the flame at low Damköhler numbers and ambient temperatures below the adiabatic flame temperature. For sufficiently low values of the ambient temperature, an intermediate regime exists in which the enhancement of the vaporization rate is due only to the fraction of the vaporized fuel decomposed at a thin reaction zone, not far from the droplet, where the temperature reaches its maximum value; the remaining fuel is decomposed very far from the droplet.
\end{abstract}

\section{Introduction}

THE QUASI-STEADY decomposition of monopropellant droplets in stagnant atmospheres has been widely studied in the past (Williams, 1965; Rosner, 1971). Lorell and Wise (1955) carried out a numerical analysis of the adiabatic decomposition of hydrazine droplets. Williams (1959) obtained more general numerical and approximate analytical solutions of the equations describing droplet decomposition with variable transport properties.

Spalding and Jain (1959) introduced the thin flame approximation for the analysis of droplet decomposition in the adiabatic case. A spherical premixed flame, of small thickness compared with its stand-off distance from the droplet surface, was positioned at a radius where the radial mass flux was equal to the mass flux through a one-dimensional premixed flame. Later Adler and Spalding (1961) introduced the idea that, for premixed reactants, if the activation energy of the reaction is sufficiently large a thin reaction zone separates a region of frozen 
flow from a region of equilibrium flow; the propagation velocity, or position of the reaction zone, can only be determined from an analysis of the reaction zone structure. The analysis by Adler and Spalding of the reaction zone structure is a worthwhile first step but incorrect for non-adiabatic cases because they neglect the total enthalpy changes across the reaction zone when evaluating the reaction rates.

Jain (1963) and Jain and Kumar (1969) used the results of Adler and Spalding to study the non-adiabatic decomposition of monopropellant droplets. Jain and Ramani (1969) have recently used this approximation to evaluate variable property effects.

Tarifa, Notario and Moreno (1962) have developed an approximate method, in which they divide the flow field in two transport regions separated by a third region of finite thickness, where complete decomposition of the fuel takes place. They use an integral method to obtain an approximate solution for the equations in the reaction region, and they patch this solution to that corresponding to the transport regions. Good agreement was found between their approximate results and the numerical solution of the equations. They obtained, in some cases, $S$ shaped curves for the vaporization rate as a function of the droplet radius or pressure, and related this fact to flame ignition and extinction.

Fendell $(1965,1969)$ has contributed, to the description of the nearly frozen and near-equilibrium regimes of monopropellant droplet decomposition, for zero activation energy, with asymptotic analysis for low and large values of the Damköhler number, or ratio between the characteristic mechanical and chemical times.

The purpose of this study is to develop and asymptotic theory for the quasi-steady decomposition of a monopropellant droplet in a quiescent atmosphere, when the gas decomposition reaction is of the Arrhenius type, with an activation temperature $E / R$ much larger than the characteristic gas temperature. If an Arrhenius rate is applicable to the gas phase decomposition, the activation temperature will be in most cases one order of magnitude larger than the maximum gas temperature. Therefore, one may expect that the asymptotic solution of the conservation equations, in the limit of large activation temperatures, will describe very accurately the characteristics of the droplet decomposition process.

In order to obtain, in the limit of infinite activation temperature, a finite enhancement of the droplet vaporization rate due to chemical reaction effects, one must assume that the pre-exponential rate constant-or the Damköhler numberis a function of the nondimensional activation temperature, growing to infinity with the activation temperature in such a way as to obtain any desired vaporization rate.

With increasing values of vaporization rate above the frozen flow values we pass from:

(i) a nearly frozen regime, with incipient effects of the chemical reaction which are responsible for the ignition conditions for sufficiently large Damköhler numbers, to

(ii) a possible intermediate partial decomposition regime, with a thin reaction 
zone not far from the droplet surface where only a fraction of the fuel is decomposed, while the remaining fuel decomposes in an extended reaction zone very far from the droplet surface, and

(iii) a third regime with a thin reaction zone where, in the limit of infinite activation energy, complete decomposition of the fuel takes place. This reactive diffusive region is separated from the droplet surface by a transport region. Only in the limit of very large Damköhler numbers, when the reaction zone is established close to the droplet surface, does the structure of these two regions become identical to that of a planar premixed flame, in conformance with the thin flame approximation of Spalding and Jain (1959).

As a result from this asymptotic analysis, simple analytical relations are obtained for the droplet vaporization rate in terms of the heats of vaporization and decomposition, the surface or boiling temperature, temperature at infinity, reaction rate constants, and transport coefficients. In particular, simple analytical expressions will be obtained for the ignition and extinction Damköhler numbers.

Although we shall assume in this presentation a constant specific heat, equal diffusivities of heat and mass, and a diffusion coefficient proportional to temperature, the theory may be easily generalized to more realistic transport coefficients as well as to more complex physical situations, as will become clear from the results. For example, the same method and ideas will be applicable to the analysis of droplet decomposition under forced convection at large Reynolds numbers, the present analysis being, of course, directly applicable to the low number Reynolds flow past a decomposing monopropellant droplet.

One should mention that the multiplicity of the decomposition regimes found here, as in earlier studies, is associated with the quasi-steady assumption. In an unsteady treatment of the gas phase decomposition the governing equations would be parabolic, and the solution could, therefore, be uniquely related to the initial conditions. Nevertheless, the background offered by a systematic analysis of the quasi-steady regimes will be essential to the transient analysis.

A stability analysis would very likely show that the regimes corresponding to the segment of the curve giving increasing vaporization rates for decreasing Damköhler numbers are not stable.

In the section on Formulation, the mathematical problem will be formulated, and it will be shown how, in the limit of large activation energies, regions of near equilibrium flow or frozen flow may coexist in the flow field. In the next section, the nearly frozen ignition regime is analyzed. The complete decomposition and the partial decomposition regimes are presented in subsequent sections. The final section is devoted to a discussion and generalization of the results.

\section{Formulation}
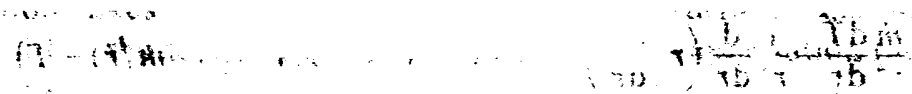

The conservation equations governing the quasi-steady gas phase distribution of fuel and energy are, in non-dimensional form,

$$
\quad \frac{\dot{m}}{r^{2}} \frac{\mathrm{d} Y}{\mathrm{~d} r}-\frac{1}{r^{2}} \mathrm{~d} d\left(r^{2} \frac{\mathrm{d} Y}{\mathrm{~d} r}\right)=-D_{1} Y \exp \left(-T_{a} / T\right)
$$




$$
\frac{\dot{m}}{r^{2}} \frac{\mathrm{d} T}{\mathrm{~d} r}-\frac{1}{r^{2}} \frac{\mathrm{d}}{\mathrm{d} r}\left(r^{2} \frac{\mathrm{d} T}{\mathrm{~d} r}\right)=D_{1} Y \exp \left(-T_{a} / T\right)
$$

The boundary conditions are

at

$$
\begin{gathered}
r \rightarrow \infty: \quad T=T_{\infty}, \quad Y=0 \\
r=1 \quad \mathrm{~d} T / \mathrm{d} r=\dot{m} L \quad \text { and } \quad \mathrm{d} Y / \mathrm{d} r=\dot{m}(Y-1), \quad T=T_{s} .
\end{gathered}
$$

We use as a boundary condition $T=T_{s}$, instead of the Clausius-Clapeyron relation between the surface concentration of fuel and surface temperature. Without loss of accuracy, one may equate $T_{s}$ to the boiling temperature, for temperatures at infinity or flame temperatures larger than the boiling temperature, and moderately large values of the heat of vaporization.

In this system of equations the radius $r$ has been made non-dimensional with the droplet radius $a, Y$ is the fuel mass fraction, $T$ the temperature, $T_{\infty}$ the temperature at infinity, and $T_{s}$ and $T_{a}$ the surface temperature and activation temperature. All temperatures have been made nondimensional with $Q / c_{p}$ where $Q$ is the heat release per unit mass of fuel, and $c_{p}$ is the specific heat at constant pressure for the gas. The heat of vaporization $L$ has been made nondimensional by dividing by the specific heat release $Q$ of the fuel. The mass transfer rate $\dot{m}$ has been made nondimensional by dividing by $4 \pi a \rho D$, where $\rho D$, which we assume to be constant, is the product of density and diffusion coefficient for the gas, $\dagger D_{1}$ is the second Damköhler number $a^{2} B / D_{\infty}$, and we have assumed a mass rate of fuel consumption per unit volume given by $\rho_{\infty} Y B \exp \left(-T_{a} / T\right)$ where $B$ is a frequency factor.

From eqns (1) and (2) and the boundary conditions (3) we deduce

$$
Y+T=T_{e}+\left(T_{\infty}-T_{e}\right) \exp (-\dot{m} / r)
$$

where $T_{e}$ is the adiabatic flame temperature,

$$
T_{e}=1-L+T_{s}
$$

The adiabatic case corresponds to $T_{\infty}=T_{e}$. For $T_{\infty}>T_{e}$ heat comes from infinity to increase the vaporization rate. For $T_{\infty}<T_{e}$ heat is lost from the reaction region to the surrounding atmosphere by conduction.

In view of eqn (4), the energy equation (2) may now be written as

$$
\frac{\dot{m}}{r^{2}} \frac{\mathrm{d} T}{\mathrm{~d} r}-\frac{1}{r^{2}} \frac{\mathrm{d}}{\mathrm{d} r}\left(r^{2} \frac{\mathrm{d} T}{\mathrm{~d} r}\right)=D_{1}\left\{T_{e}+\left(T_{\infty}-T_{e}\right) \exp (-\dot{m} / r)-T\right\} \exp \left(-T_{a} / T\right)
$$

to be solved with the boundary conditions 


$$
\begin{gathered}
r \rightarrow \infty: \quad T=T_{\infty} \\
r=1: \quad T=T_{s} \quad \text { and } \quad \mathrm{d} T / \mathrm{d} r=\dot{m} L .
\end{gathered}
$$

Because of the three boundary conditions, the solution of (6) is possible only for definite values of $\dot{m}$, which then appears as an eigen-value of this problem. We shall attempt to obtain the asymptotic solution of (6) with the boundary conditions (7) and (8), for large values of the activation temperature $T_{a}$, assuming $T_{s}, T_{\infty}$, and $L$, and therefore $T$, to be of order unity. If in (6) we take the limit $T_{a} \rightarrow \infty$ we find the following two limiting equations, depending on our choice of $D_{1}\left(T_{a}\right)$ :

(a) Frozen flow described by the equation

$$
\frac{\dot{m}}{r^{2}} \frac{\mathrm{d} T}{\mathrm{~d} r}-\frac{1}{r^{2}} \frac{\mathrm{d}}{\mathrm{d} r}\left(r^{2} \frac{\mathrm{d} T}{\mathrm{~d} r}\right)=0
$$

with the general solution,

$$
T=A_{1}+A_{2} \exp (-\dot{m} / r)
$$

where $A_{1}$ and $A_{2}$ are integrating constants, and

(b) Equilibrium flow given by $Y=0$, or

$$
T=T_{e}+\left(T_{\infty}-T_{e}\right) \exp (-\dot{m} / r)
$$

Uniformly valid asymptotic solutions, for large $T_{a}$, of (6) with the boundary conditions (7) and (8) have the limiting general form (10), that correspond to frozen flow, or the particular form (11), depending on the region of the flow field and on the value of the Damköhler number. We shall see that there are cases in which the solution of (6)-(8) is multivalued in a given range of $D_{1}$, thereby exhibiting the ignition and extinction characteristics. The following regimes are possible:

We anticipate that a weak mode of decomposition, or ignition regime, will exist for which the mass rate of vaporization will not deviate much from the frozen-flow or pure-vaporization value. The temperature will be given in the first approximation by the frozen flow solution (10) satisfying the boundary conditions (7) and (8). This is because, due to the small values of the reaction rate, most of the fuel decomposition takes place so far from the droplet surface that the heat release affects neither the temperature distribution nor the vaporization rate, in a first approximation.

An intermediate mode of decomposition, or partial decomposition regime, may exist for which part of the fuel burns at a thin reaction region, not far from the droplet surface, where the temperature rises above the temperature at infinity so that an enhancement of the vaporization rate occurs. The temperature drops on both sides of this reaction region and, because of the large activation temperature, 
the chemical reaction is practically frozen. The asymptotic solution has the form (10) on both sides of the thin reaction region; the constants $A_{i}$ are determined from the boundary conditions (7) and (8) and matching conditions with the solution appropriate to the thin reaction region. The rest of the fuel is then decomposed only far from the droplet, with negligible effects on the vaporization rate, as described by the nearly frozen solution of Fendell (1965). This mode of decomposition is possible only for $T_{\infty}$ sufficiently below $T_{e}$, and large activation temperatures.

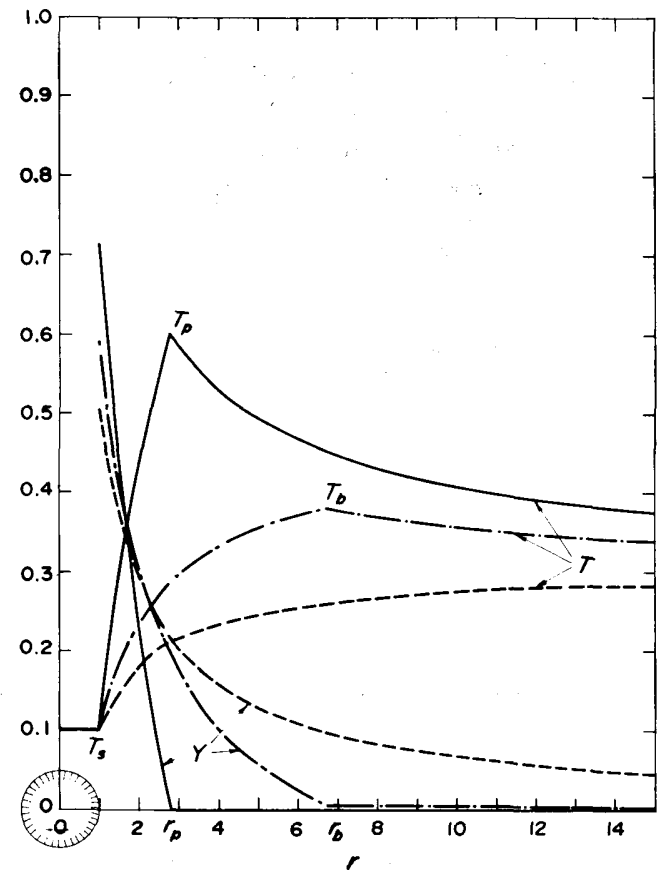

Fig. 1. The temperature and fuel mass fraction distributions, in the limit of infinite activation energy, for the three decomposition regimes: _ _ complete decomposition; -.-.-, partial decomposition; ------, frozen decomposition.

In a third mode of burning, which we shall term the complete decomposition regime, most of the fuel is descomposed at a reaction region which is thin because of the large activation temperature. The small amount of fuel crossing the thin reaction region burns only very far from the droplet. Then the asymptotic solution is of the form (10), satisfying the boundary conditions (8), inside the thin reaction zone, and is given by (11) outside of the reaction zone. The reaction zone location is determined by matching these two limiting solutions to the appropriate thin reaction zone solution. In Fig. 1 the temperature distribution is sketched for the three regimes. 
Frozen flow with pure vaporization

Assuming, in what follows, that $T_{\infty}>T_{s} \dagger$, we shall write now, for future reference, the results corresponding to pure vaporization with frozen flow. The Damköhler number, referred to the conditions at infinity, $D_{1} \exp \left(-T_{\infty} / T_{a}\right)=D_{\infty}$, must be small enough so that the chemical reaction may be neglected in the first approximation for $r \ll D_{\infty}{ }^{-1 / 2}$; the first approximation for the temperature distribution is given by

$$
\frac{\dot{m}_{o}}{r^{2}} \frac{\mathrm{d} T_{o}}{\mathrm{~d} r}-\frac{1}{r^{2}} \frac{\mathrm{d}}{\mathrm{d} r}\left(r^{2} \frac{\mathrm{d} T_{o}}{\mathrm{~d} r}\right)=0
$$

with the boundary conditions

$$
\begin{gathered}
r \rightarrow \infty \quad T_{o}=T_{\infty} \\
r=1 \quad T_{o}=T_{s} \quad \text { and } \quad \mathrm{d} T_{o} / \mathrm{d} r=\dot{m}_{o} L .
\end{gathered}
$$

The solution is

$$
T_{o}=-L+L \exp \left\{m_{o}(1-1 / r)\right\}+T_{s}
$$

and

$$
\dot{m}_{o}=\ln \left\{\left(T_{\infty}-T_{s}+L\right) / L\right\}
$$

which is the decomposition rate corresponding to pure vaporization.

For large values of $r, T_{o}$ decays as

$$
T_{o}=T_{\infty}-\mu / r, \text { where } \mu=\left(T_{\infty}-T_{s}+L\right) \dot{m}_{o} .
$$

A regular expansion of $T$, for small values of $D_{\infty}$, is not uniformly valid, because of the singular perturbation character of the problem, as shown by Fendell (1965) for zero activation energy. This problem may be solved, for moderate activation energies, by the method of matched asymptotic expansions, using $D_{\infty}$ as the small parameter, with a procedure identical to that used by Fendell (1965) for the case of zero activation energy. It is not difficult to show that the first two terms of the inner expansion and the first term of the outer expansion, obtained by Fendell, are exact for moderate energies of activation different from zero, if the reaction rate constant is evaluated at the temperature at infinity. For activation temperatures large compared with $T_{\infty}$, ignition and extinction characteristics occur, that cannot be analyzed by expansions in small or large $D_{\infty}$, because they become divergent.

\section{Near ignition regime}

We shall now analyze the near ignition regime; that is, we shall look for the asymptotic solution of eqn (6), for small values of $\epsilon=T_{\infty}{ }^{2} / T_{a}$, and Damköhler

$\nmid$ Pure vaporization can not take place under quasi-steady conditions unless $T_{\infty}>T_{s}$. We could relax this condition when chemical reaction occurs. 
numbers $D_{1}$ such that the temperature increment above the frozen flow value is of order $T_{\infty}{ }^{2} / T_{a}$, so that the non-linear effects associated with the Arrhenius exponent are important.

The resulting decomposition rate

$$
\dot{m}=\dot{m}_{o}+\epsilon \dot{m}_{1}
$$

rises above the vaporization value $\dot{m}_{o}$ by a quantity of order $\epsilon$. The problem we pose is to find the asymptotic solution of eqn (6) and the Damköhler number producing a given increment $\epsilon \dot{m}_{1}$ of the decomposition rate above $\dot{m}_{o}$.

A two-zone structure emanates from an asymptotic analysis of eqn (6) for small $\epsilon$. In an inner transport zone, for $r$ of order unity, the chemical reaction is exponentially small due to the presence of the Arrhenius exponent. This becomes clear from eqn (6), when written in the form

$$
\begin{gathered}
\frac{\dot{m}}{r^{2}} \frac{\mathrm{d} T}{\mathrm{~d} r}-\frac{1}{r^{2}} \frac{\mathrm{d}}{\mathrm{d} r}\left(r^{2} \frac{\mathrm{d} T}{\mathrm{~d} r}\right)=D_{\infty}\left\{T_{e}+\left(T_{\infty}-T_{e}\right) \exp (-\dot{m} / r)-T\right\} \exp \left\{\left(T_{a} / T_{\infty}\right)\right. \\
\left.\times\left(T-T_{\infty}\right) / T\right\} .
\end{gathered}
$$

If in eqn (17) we take the limit $\left(T_{\infty} / T_{a}\right) \rightarrow 0$, anticipating an algebraic variation with $T_{a}$ of the factor $D_{\infty}$, then the chemical reaction becomes exponentially frozen for $T<T_{\infty}$, not so close to $T_{\infty}$ as to make $\left(T-T_{\infty}\right)$ of order $T_{\infty}{ }^{2} / T_{a}$.

The temperature is then given, to all algebraic orders in $\epsilon$, by the frozen flow value

$$
T=T_{s}-L+L \exp \left\{\left(\dot{m}_{o}+\epsilon \dot{m}_{1}\right)(1-1 / r)\right\}
$$

which satisfies the interior boundary conditions (8). The inner expansion may be obtained by expanding the temperature given by (18) in powers of $\epsilon$, for fixed $r$ of order unity. The solution (18) ceases to be valid for those large values of $r$ for which the resulting temperature is so close to $T_{\infty}$ as to make $T-T_{\infty} \sim T_{\infty}{ }^{2} / T_{a}$.

For the outer reaction zone, we introduce the outer variable

$$
\xi=r \epsilon \text {, }
$$

the expansion

$$
T=T_{\infty}+\epsilon \theta_{1}+\epsilon^{2} \theta_{2}+\cdots
$$

where $\theta_{1}, \theta_{2}, \ldots$ are functions of $\xi$, and the expansion

$$
\Delta=\Delta_{o}+\epsilon \Delta_{1}+\cdots
$$

of the reduced Damköhler number

$$
\Delta=\left(T_{a}^{2} / T_{\infty}^{4}\right) D_{1} \exp \left(-T_{a} / T_{\infty}\right)
$$


We anticipate that $\Delta_{o}$ will be of order unity in the near-ignition regime.

By taking the expansions (20) and (21) into eqns (6) or (17), previously written in term $\xi$, we obtain the following equation for $\theta_{1}$

$$
\xi^{-2} \frac{d}{d \xi}\left(\xi^{2} \frac{d \theta_{1}}{d \xi}\right)=\Delta_{o}\left(\theta_{1}+\alpha \mu / \xi\right) \exp \theta_{1}
$$

where

$$
\alpha=\left(T_{\infty}-T_{e}\right) /\left(T_{\infty}-T_{s}+L\right)=\left(T_{\infty}-T_{e}\right) /\left(1+T_{\infty}-T_{e}\right) .
$$

Equation (23) represents a balance of the diffusion and chemical reaction effects; convective effects do not appear in the first approximation. In addition, the Arrhenius exponent has been linearized about $T_{\infty}$.

In order to facilitate the numerical integration of eqn (23), we shall write it in terms of the variables

$$
y=-\theta_{1} \xi / \mu \text { and } x=\sqrt{\Delta_{o}} \xi
$$

so that we obtain the equation

$$
d^{2} y / d x^{2}=(y-\alpha) \exp \left(-\mu \sqrt{\Delta_{o}} y / x\right)
$$

to be solved with the boundary conditions

$$
y(\infty)=\alpha, \quad y(0)=1 .
$$

The last boundary condition is obtained from the matching conditions with the inner solution (10). In fact, when matching the outer expansion (20) and the inner solution (18) in an intermediate variable $\xi_{i}=r \epsilon^{i}$ with $0<i<1$, we obtain,

$$
\begin{aligned}
T & =T_{\infty}-\epsilon^{i} y(0) \mu / \xi_{i}-\epsilon \sqrt{\Delta_{o}} \mu y^{\prime}(0)+O\left(\epsilon^{2-i}\right) \\
& =T_{s}-L+\left(L \exp \dot{m}_{o}\right)\left\{1-m_{o} \epsilon^{i} / \xi_{i}+\dot{m}_{1} \epsilon+O\left(\epsilon^{2 i}\right)\right\} .
\end{aligned}
$$

By identifying the terms of order unity in eqn (28) we obtain eqn (14); by identifying the terms of order $\epsilon^{i}$, we obtain the boundary condition $y(0)=1$. Matching terms of order $\epsilon$ gives

$$
\dot{m}_{1} L \exp \dot{m}_{o}=-\mu \sqrt{\Delta_{o}} y^{\prime}(0)
$$

where the derivative $y^{\prime}(0)$ is a function of $\mu \sqrt{\Delta_{o}}$ and $\alpha$, to be determined from the solution of eqn (26). This equation was solved numerically, and the resulting values of $\mu \sqrt{\Delta_{o}} y^{\prime}(0)$ are plotted in Fig. 2 in terms of $\mu \sqrt{\Delta_{o}}$ for several values of $\alpha$.

The relation (29) gives, in fact, a first approximation for the enhancement of 


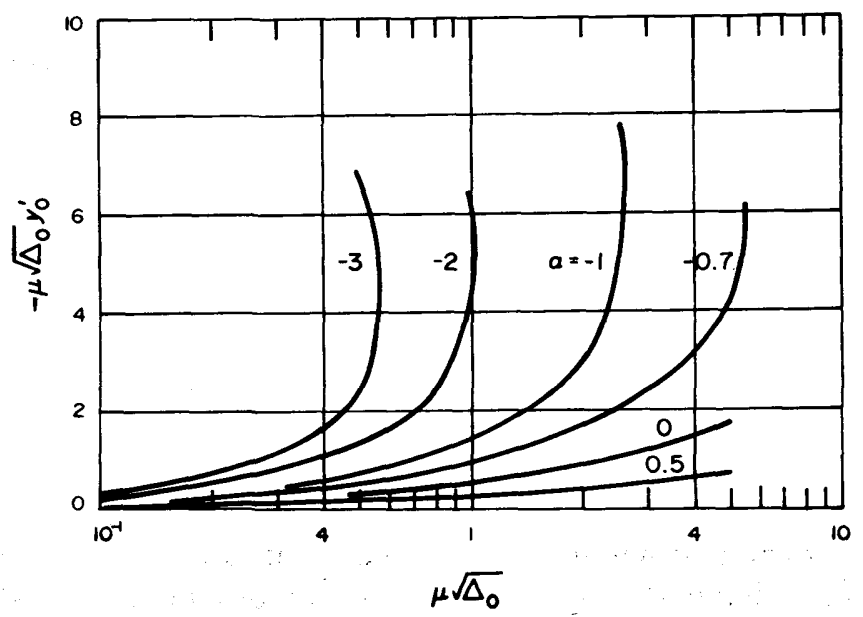

Fig. 2. Enhancement of the vaporization rate, in the nearly frozen regime, as a function of the reduced Damköhler number, $\Delta_{o} \mu^{2}$, for several values of the parameter $\alpha=$ $\left(T_{\infty}-T_{e}\right) /\left(1+T_{\infty}-T_{e}\right)$.

the droplet rate of decomposition in terms of the Damköhler number. It is clear from Fig. 2 that the rate of vaporization is a multivalued function of the Damköhler number for negative values of $\alpha$, that is, for an ambient atmosphere with temperature $T_{\infty}$ lower than the adiabatic flame temperature. If $\alpha$ is negative, no solution of eqn (26) exists for values of $\mu \sqrt{\Delta_{o}}$ above an "ignition" value $\mu \sqrt{\Delta_{o I}}$ which is a function of $\alpha$, represented in Fig. 3 .

An approximate expression for $\mu \sqrt{\Delta_{o l}}$ in terms of $\alpha$ is

$$
\mu \sqrt{\Delta_{o}} \alpha^{2}=16 e^{-2} / \sqrt{2}-\alpha=1.531-\alpha
$$

which has the correct asymptotic form for low values of $(-\alpha)$, and for large values

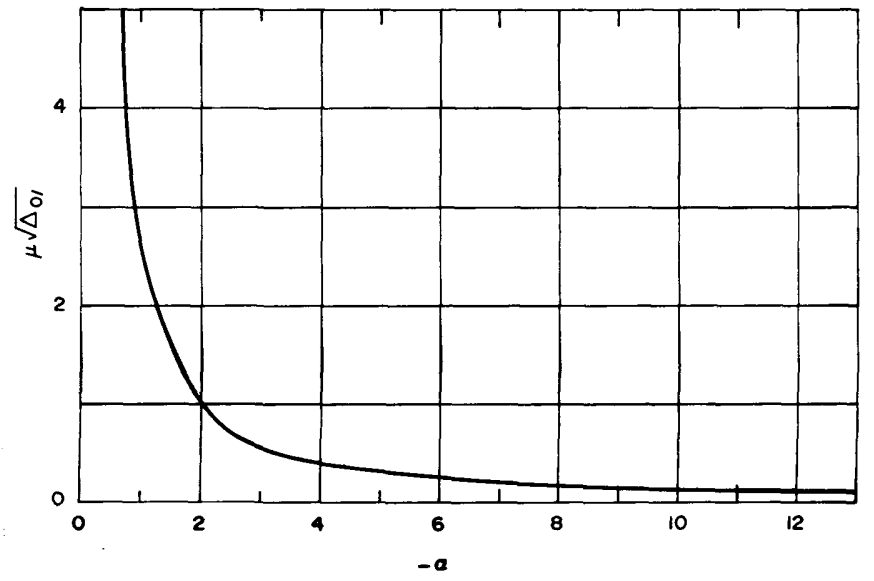

Fig. 3. The reduced Damköhler number of ignition as a function of the parameter $\alpha$. 
of $-\alpha$, if, according to our numerical calculations the term $-\alpha$ is substituted by $-0.998 \alpha$.

When $\alpha$ approaches zero from below, $\mu \sqrt{\Delta_{\text {ol }}}$ goes to infinity; the chemical reaction occurs at small values of $\xi$, with large deviations of the temperature from $T_{\infty}$, so that the expansion (20) cannot be used. However, for these small values of $\alpha$, the chemical reaction occurs in a thin transition zone from the region of frozen flow to the region of equilibrium; the analysis of the complete decomposition regime given in the next section may then be used to describe the ignition conditions.

As it has been indicated before, there are two solutions of eqn (23) for negative values of $\alpha$. The vaporization rate is higher, and increases with decreasing values of $\mu \sqrt{\Delta_{o}}$, in one of the solutions, which thus corresponds very likely to an unstable mode of decomposition. For small values of $\mu \sqrt{\Delta_{o}}$ this solution shows a thin chemical reaction separating an interior region of frozen flow from an outer region in near equilibrium if $\alpha>-1$, or frozen flow if $\alpha<-1$ when complete decomposition occurs only very far from the droplet. The asymptotic form of this solution for small values of $\mu \sqrt{\Delta_{o}}$ can be obtained by the analyses of the following sections for the complete and partial decomposition regimes.

For positive values of $\alpha$, the relation between the droplet vaporization rate and Damköhler number is single-valued, so that no clearcut ignition conditions are obtained in this case.

\section{Complete decomposition regime}

For large activation temperatures and sufficiently large Damköhler numbers, an interior region of frozen flow and an outer region of near-equilibrium flow are separated by a thin reaction zone. The asymptotic analysis presented here shows how the approximate procedure of Tarifa et al. (1962) who divide the flow field in three similar regions, is correct for large activation energies.

For cold atmospheres, i.e. negative $\alpha$, the temperature reaches its maximum value in the reaction region. The reaction time is, at this temperature, of the order of the diffusion time through the reaction zone, and nearly complete decomposition of the fuel takes place there. In the outer region the reaction time, due to the lower temperature, becomes exponentially large compared with the diffusion time except at very large distance from the droplet. For hot atmospheres, $\alpha \geq 0$, the temperature increases from the reaction zone to infinity, and complete equilibrium is reached on crossing the reaction zone, therefore, without additional chemical reaction effects.

In order to see this more clearly, let us assume that the flame temperature is $T_{p}$ in the first approximation, and write eqn (6) in the form

$$
\begin{aligned}
\frac{\dot{m}}{r^{2}} \frac{\mathrm{d} T}{\mathrm{~d} r}-\frac{1}{r^{2}} \frac{\mathrm{d}}{\mathrm{d} r}\left(r^{2} \frac{\mathrm{d} T}{\mathrm{~d} r}\right)= & \left\{T_{e}+\left(T_{\infty}-T_{e}\right) \exp (-\dot{m} / r)-T\right\} D_{1} \exp \left(-T_{a} / T_{p}\right) \\
& \times \exp \left\{T_{a}\left(T-T_{p}\right) / T_{p} T\right\} .
\end{aligned}
$$

If we anticipate that 


$$
D_{1} \exp \left(-T_{a} / T_{p}\right) \sim T_{a}^{2},
$$

by taking the limit $T_{a} \rightarrow \infty$ in eqn (30), we obtain frozen flow for $\left(T_{p}-T\right) \gg T_{p}^{2} / T_{a}$, and equilibrium flow for $\left(T-T_{p}\right) \gg T_{p}^{2} / T_{a}$. The chemical reaction will only be important at temperatures $T-T_{p} \sim T_{p}^{2} / T_{a}$. However, we claim that even for $\alpha<0$, when $T$ decreases below $T_{p}$ in the outer frozen flow region, we have there equilibrium flow in the first approximation, because nearly complete decomposition of the fuel took place at the thin reaction zone.

We shall now look for the Damköhler number and the asymptotic solution of eqn (30), for large values of $T_{a}$, for which the droplet decomposition rate is a given value $\dot{m}$.

We have in the first approximation an interior frozen flow region where the temperature is given by

$$
T=T_{s}-L+L \exp (\dot{m}-\dot{m} / r)
$$

which is of the form (10), and satisfies the boundary conditions at the droplet surface (8). The corresponding fuel concentration in this region may be obtained from eqns (4) and (32), as

$$
Y=1+\left(T_{\infty}-T_{e}-L \exp \dot{m}\right) \exp (-\dot{m} / r) .
$$

If in the outer region equilibrium, $Y=0$, occurs in the first approximation, we obtain according to eqn (4) the following temperature distribution

$$
T=T_{e}+\left(T_{\infty}-T_{e}\right) \exp (-\dot{m} / r) .
$$

These two regions are separated, in the limit $T_{a} \rightarrow \infty$, by an infinitely thin reaction zone at $r=r_{p}$, where the temperature is $T_{p}$, as obtained from eqns (32) and (34) at $r=r_{p}$; that is

$$
T_{p}=T_{s}-L+L \exp \left(\dot{m}-\dot{m} / r_{p}\right)
$$

and

$$
T_{p}-T_{e}=\left(T_{\infty}-T_{e}\right) \exp \left(-\dot{m} / r_{p}\right) .
$$

The droplet vaporization rate $\dot{m}$ is then related to the flame temperature $T_{p}$ by

$$
\dot{m}=\ln \left\{\left(T_{p}-T_{s}+L\right)\left(T_{\infty}-T_{e}\right) /\left(T_{p}-T_{e}\right) L\right\} .
$$

To obtain the relation between the Damköhler number and the vaporization rate, we shall use the matching conditions between an inner expansion for the temperature within the reaction zone and the expansions for the interior and outer zones. We shall use $T_{p}{ }^{2} / T_{a}=\epsilon$ as the small parameter, where $T_{p}$ is given by eqn (37) in terms of $\dot{m}$. 
Anticipating that in the interior region the chemical reaction will be frozen to all algebraic orders in $\epsilon$, the temperature will be given in this region by eqn (32).

For the outer region we use the expansion

$$
T=T_{e}+\left(T_{\infty}-T_{e}\right) \exp (-\dot{m} / r)-\{1-\exp (-\dot{m} / r)\}\left(\epsilon P_{1}+\epsilon^{2} P_{2}+\cdots\right)
$$

which satisfies the outer boundary condition (7).

For the reaction zone we introduce the expansion

$$
T=T_{p}-\epsilon\left\{\left(T_{e}-T_{p}\right) \eta+p_{o}+y_{1}\right\}-\epsilon^{2} y_{2}+\cdots
$$

where $y_{1}, y_{2}, \ldots$ are functions of the inner variable $\eta$,

$$
\eta=\dot{m}\left(r-r_{p}\right) / \epsilon r_{p}^{2}-p_{o} /\left(T_{e}-T_{p}\right),
$$

and $p_{o}$, which we anticipate to be of order unity, is the first term of an expansion in $\epsilon$

$$
p=p_{o}+\epsilon p_{1}+\cdots
$$

of

$$
p=\ln \left\{\left(T_{p}^{2} r_{p}^{2} / \dot{m} T_{a}\right)^{2} 2 D_{1} \exp \left(-T_{a} / T_{p}\right)\right\}
$$

The inner variable $\eta$ and the form of $p$ have been chosen so as to obtain for $y_{1}(\eta)$ the equation

$$
2 \mathrm{~d}^{2} y_{1} / \mathrm{d} \eta^{2}=y_{1} \exp \left\{-y_{1}-\left(T_{e}-T_{p}\right) \eta\right\}
$$

and the boundary conditions

$$
\mathrm{d} y_{1} / \mathrm{d} \eta=0 \text { for } \eta \rightarrow \infty, \quad \mathrm{d} y_{1} / \mathrm{d} \eta=-1 \text { for } \eta \rightarrow-\infty .
$$

These boundary conditions are obtained from the matching conditions with the outer solutions, eqns (32) and (38). The choice of the factor 2 in eqn (43) was made so that a solution of this equation with the boundary conditions (44) exists in the limiting case $T_{e}-T_{p}=0$.

Equation (43) shows that, in the first approximation, the heat evolved by the chemical reaction is conducted, without convective effects toward both sides of the reaction zone. The exponential factor $\exp \left(T_{p}-T_{e}\right) \eta$ represents the effect on the reaction rate of the heat loss from the reaction zone toward the nearequilibrium side of the flame. Aside from this factor the structure of the reaction zone would be identical to that of an ordinary pre-mixed flame, as analyzed, for example, by Bush and Fendell (1970, 1972).

Equation (43) describes also the reaction zone structure in one of the combustion regimes of quasi-steady diffusion flames (Liñán, 1974). It has been 
solved numerically, with the boundary conditions (44), so as to obtain $y_{1}(\eta)$ for several values of $\left(T_{e}-T_{p}\right)$. The limiting values $y_{1 \infty}$ and $n$,

$$
y_{1 \infty}=\lim _{\eta \rightarrow \infty} y_{1}, \quad n=\lim _{\eta \rightarrow-\infty}\left(y_{1}+\eta\right)
$$

were obtained by Liñán (1974) as a function of $\left(T_{e}-T_{p}\right)$. The following expression was found to correlate well the numerical results for the relation between $n$ and $u=T_{e}-T_{p}$, in the range $-0 \cdot 2<u<0 \cdot 5$ :

$$
n=1.344-4 u(1-u)(1-2 u)+3 u^{2}-u^{-1} \ln \left(1-4 u^{2}\right) .
$$

There is no solution of eqns (43) and (44) for values of $\left(T_{e}-T_{p}\right)>0.5$; this means that in order to have a premixed flame regime the temperature given by the thin flame solution (eqns 32 and 34), must decrease at a faster rate toward the droplet surface than toward the outer near-equilibrium region.

The matching conditions of the two term inner expansion (39) with the interior solution (32) and with the two term outer expansion (38) provide, in addition, the following relations.:

$$
P_{1}\left\{1-\exp \left(-\dot{m} / r_{p}\right)\right\}=y_{1 \infty}
$$

and

$$
p_{o}=-n\left(T_{e}-T_{p}\right)
$$

The first relation enables us to calculate the distribution in the outer region of the fuel leaking through the flame, which is proportional to $\epsilon y_{1 \infty}$.

Equation (47) gives the first approximation for the Damköhler number in terms of the flame temperature $T_{p}$. That is,

$$
2 D_{1}\left(T_{p}^{2} r_{p}^{2} / \dot{m} T_{a}\right)^{2} \exp \left(-T_{a} / T_{p}\right)=\exp \left\{-n\left(T_{e}-T_{p}\right)\right\}
$$

Equation (48) together with eqns (36), (37), and (45) give an explicit parametric representation of the relation between the Damköhler number and the vaporization rate $\dot{m}$.

It is possible to show, by using singular perturbation methods, that when $T_{e}-T_{p}$ approaches 0.5 the leakage of the fuel through the reaction zone increases toward infinity,

$$
y_{1 \infty} \doteq\left\{0.5-\left(T_{e}-T_{p}\right)\right\}^{-1}
$$

For positive values of $\left(T_{p}-T_{e}\right)$, that is for hot atmospheres $T_{\infty}>T_{e}$, the leakage of fuel is zero; i.e. $y_{1 \infty} \equiv 0$. It can be shown that for large positive values of $\left(T_{p}-T_{e}\right), n\left(T_{e}-T_{p}\right)$ has the asymptotic form, $n\left(T_{e}-T_{p}\right) \doteq \ln \left\{2\left(T_{p}-T_{e}\right)^{2}\right\}-2 \gamma_{E}$, where $\gamma_{E}$ is the Euler constant $\gamma_{E}=0.577$. For small values of $\left(T_{e}-T_{p}\right)$, $n=1.344$.

Notice that for hot atmospheres, $T_{\infty}>T_{e}$, the range of possible values of 
$T_{e}-T_{p}$ is from $T_{e}-T_{\infty}$ to zero, remaining always negative, so that we have equilibrium flow, to all algebraic orders in $\epsilon$, outside of the reaction zone. That is, $P_{1}=P_{2}=\cdots=0$. Equation (48) may be used to determine, in the first approximation, the Damköhler number for all values of $T_{p}$ not too close to $T_{\times}$, or equivalently, for all values of $\dot{m}$ not too close to $\dot{m}_{o}$. When the resulting value of $T_{p}$ is close to $T_{\infty}$, so that $T_{\infty}-T_{p}$ is of order $\epsilon$, the reaction zone ceases to be thin, and this complete decomposition analysis should be replaced by the near-ignition analysis of the previous section.

For cold atmospheres, $T_{\infty}<T_{e}, T_{p}$ is lower than $T_{e}$, so that $T_{e}-T_{p}$ is positive, ranging from $T_{e}-T_{\infty}$ to zero whem $\dot{m}$ increases from $\dot{m}_{\circ}$ to infinity. If $T_{\infty}<$ $T_{e}-0.5$ there is a range of $\dot{m}$ between $\dot{m}_{o}$ and $\dot{m}_{t}=\ln \left\{\left(T_{e}-T_{\infty}\right) / L\right\}$ that cannot be covered by the complete decomposition analysis. This range corresponds to the partial decomposition regime that we shall analyze in the following section. If $T_{\infty}>T_{e}-0.5$, eqn (48) gives, in the first approximation, the Damköhler number in terms of $\dot{m}$ for the whole range of $\dot{m}>\dot{m}_{o}$, not so close to $\dot{m}_{o}$ that $\dot{m}-\dot{m}_{o}$ be of order $\epsilon$.

For large values of $\dot{m}$ the resulting flame temperature $T_{p}$ is close to the adiabatic flame temperature, and the reaction zone is located close to the droplet surface, $r_{p}-1 \ll 1$. Then eqn (48) reduces, in a first approximation, for large $\dot{m}$ to

$$
2 D_{1}\left(T_{e}^{2} / \dot{m} T_{a}\right)^{2} \exp \left(-T_{a} / T_{e}\right)=1 .
$$

This approximation results from neglecting the non-planar effects in the thin transport region between the droplet surface and the reaction zone, and also the effect of the heat loss by conduction from the reaction zone toward the outer transport zone.

For this approximation to be valid $\dot{m}$ must be large enough so that the value of $T_{p}$ resulting from eqn (37) is very close to $T_{e}$, that is $T_{p}-T_{e} \ll \epsilon$. The nonplanar effects in the interior transport zone as well as the heat loss from the reaction zone to the ambient atmosphere are taken into account in eqn (37) and result in $T_{n}$ being different from $T_{e}$.

A much better approximation for the Damköhler number, for moderately large $\dot{m}$, results from using eqn (48) with the exponent $n\left(T_{e}-T_{p}\right)$ substituted by 1.344 $\left(T_{e}-T_{p}\right)$. The resulting Damköhler numbers will be practically equal to those obtained by using the more exact eqn (45).

In Fig. 4 , as an example, we have plotted $\dot{m}$ in terms of $D_{1}$, for some representative values of the parameters. The extinction conditions appear very clearly in this plot.

In summarizing, eqn (48) is a relation among the Damköhler number, the flame temperature and the vaporization rate. The parameter $n$ appearing in this equation (48) is given by eqn (45) in terms of $T_{p}-T_{\infty}$, and may be taken as equal to $1 \cdot 344$, with a very small effect on the value of the logarithm of the Damköhler number. The vaporization rate and the flame temperature are related by eqn (37), and the flame location $r_{p}$ may be written in terms of $\dot{m}$ or $T_{p}$ by using eqns (36) and (37). Equation (49) shows how for large $D_{1} \dot{m} \sim a$, and thus the droplet radius decreases linearly with time. 


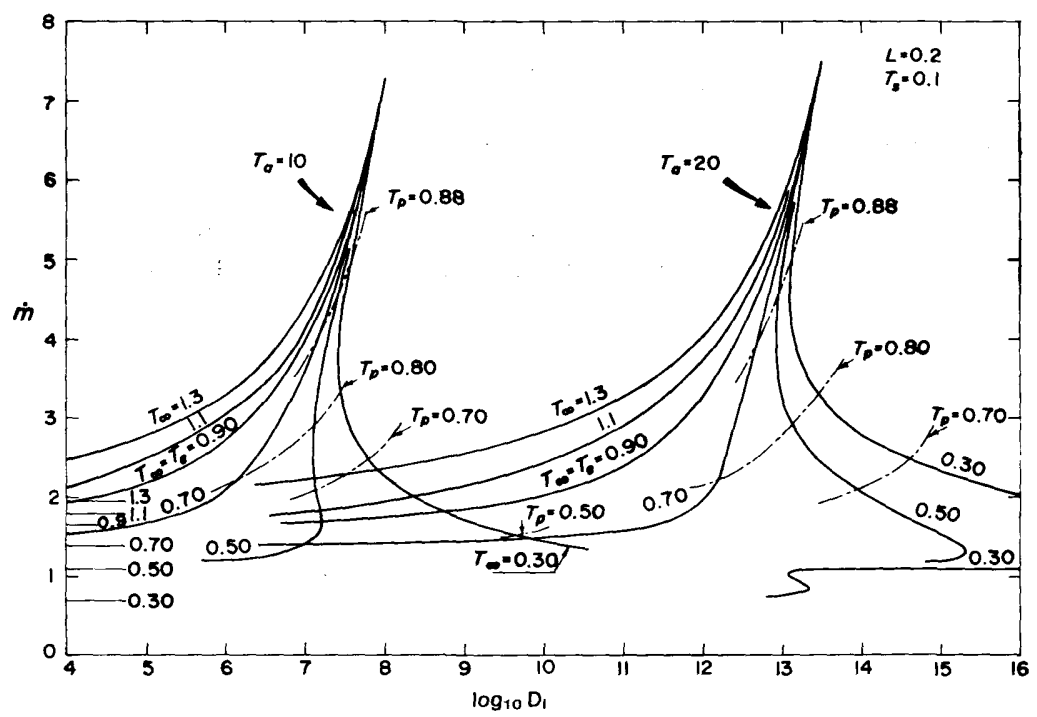

Fig. 4. Typical curves for the vaporization rate, $\dot{m}$, as a function of the Damköhler number $D_{1}$, as obtained from the analysis of the complete decomposition regime. The curve in the lower right hand side corner, for $T_{a}=10$ and $T_{\infty}=0.3$, was obtained using the theory of the partial burning regime.

\section{Partial decomposition regime}

If the temperature at infinity $T_{\infty}$ is lower than $T_{e}-0.5$, an intermediate partial decomposition regime exists for values of $\dot{m}$ lower than $\dot{m}_{t}=\ln \left\{\left(T_{e}-T_{x}\right) / L\right\}$. A thin-flame solution with complete decomposition of the fuel in the reaction zone would result in a temperature distribution that decreases at a faster rate toward infinity than toward the droplet. Then, as indicated before, there is no solution of the eqns (43) and (44) that describes the structure of the reaction zone.

We claim that in this case a fraction of the fuel flux is decomposed at a thin reaction zone, where the temperature reaches its maximum value. Outside this thin reaction zone the chemical reaction is quenched, because of the lowering of the reaction rates with decreasing temperatures. The fuel leaking through the flame will burn only at large distances from the droplet surface, without affecting the vaporization rate.

We shall now find the asymptotic solution, for large $T_{a}$, of eqns (6)-(8), and the Damköhler number that produces a vaporization rate $\dot{m}$, intermediate between $\dot{m}_{o}$ and $\dot{m}_{t}$. We anticipate that the reaction-zone thickness is of order $1 / T_{a}$, that is, infinitely thin in the limit $T_{a} \rightarrow \infty$, with a resulting flame location, $r_{b}$, and temperature, $T_{b}$, that we shall determine below.

In the limit $T_{a} \rightarrow \infty$, the temperature will be given by eqn (32) in the interior frozen flow region, $r<r_{b}$. In the outer frozen flow region, $r>r_{b}$, the temperature will be given by

$$
T-T_{\infty}=\left(T_{b}-T_{\infty}\right)\{1-\exp (-\dot{m} / r)\} /\left\{1-\exp \left(-\dot{m} / r_{b}\right)\right\} .
$$


And, according to eqn (32), the flame temperature $T_{b}$ and flame location will be related by

$$
T_{b}=T_{s}-L+L \exp \left(\dot{m}-\dot{m} / r_{b}\right)
$$

From eqn (4) we may obtain a relation between $Y_{b}, r_{b}, T_{b}$, and $\dot{m}$.

The asymptotic structure has now been completely described in term of $T_{b}$ and $\dot{m}$. The flame temperature $T_{b}$ and the Damköhler number will be determined below in terms of $\dot{m}$ by the method of matched asymptotic expansions, for small values of $\epsilon=T_{b}^{2} / T_{a}$.

The chemical reaction will be frozen, to all algebraic orders in $\epsilon$, in the regions interior and exterior to the reaction zone, because the temperature in these regions decreases below $T_{b}$ by an amount large compared with $\epsilon$. We then take eqn (32) as giving the temperature distribution in the interior region, and use the following expansion for the outer region:

$$
T-T_{\infty}=\{1-\exp (-\dot{m} / r)\}\left(1-\exp \left(-\dot{m} / r_{b}\right)\right\}^{-1}\left(T_{b}-T_{\infty}+\epsilon B_{1}+\epsilon^{2} B_{2}+\cdots\right) .
$$

For the temperature within the reaction zone we use the expansion

$$
T=T_{b}+\epsilon \Phi_{1}(\zeta)+\epsilon^{2} \Phi_{2}(\zeta)+\cdots
$$

in terms of the inner variable

$$
\zeta=\left(r-r_{b}\right) / \epsilon .
$$

We also expand the Damköhler number as

$$
D_{1}=D_{b}\left(1+\epsilon b_{1}+\cdots\right),
$$

where $D_{b}$ is the value of $D_{1}$ which, in the first approximation, makes possible the matching between the reaction zone solution and the outer solutions.

When introducing the expansion (53) in eqn (6), previously written in terms of $\zeta$, we obtain for $\Phi_{1}$ the equation

$$
\mathrm{d}^{2} \Phi_{1} / \mathrm{d} \zeta^{2}=-\Lambda_{0} \exp \Phi_{1}
$$

where

$$
\Lambda_{o}=Y_{b} D_{b}\left(T_{b}^{2} / T_{a}\right) \exp \left(-T_{a} / T_{b}\right)
$$

From eqn (56) we obtain

$$
\left(\mathrm{d} \Phi_{1} / \mathrm{d} \zeta\right)^{2}=2 \Lambda_{\circ} \exp \left(\Phi_{m}-\Phi_{1}\right)
$$


and

$$
2 \ln \left(t+\sqrt{ } t^{2}-1\right)= \pm\left(2 \Lambda_{o} \exp \Phi_{m}\right)^{1 / 2}\left(\zeta-\zeta_{m}\right)
$$

where $t=\exp \left\{\left(\Phi_{m}-\Phi_{1}\right) / 2\right\}$, and the integrating constants $\Phi_{m}$ and $\zeta_{m}$ give the maximum temperature and its location. The plus and minus sign in eqn (59) must be used for the positive and negative values of $\left(\zeta-\zeta_{m}\right)$, respectively.

We must determine $\zeta_{m}, \Phi_{m}$ and the reduced Damköhler number $\Lambda_{o}$, from the matching conditions between the inner and outer expansions. Matching to zero order in $\epsilon$ is automatic, because we anticipated that the maximum temperature given by the inner and outer expansions would be equal to $T_{b}$. Matching to an order intermediate between $\epsilon^{\circ}$ and $\epsilon$, carried out in intermediate variables, indicates that the slopes, at the outer edges of the reaction zone, of the temperature distribution given by the two-term inner expansion and the one-term outer expansions should be equal. Thus we obtain

$$
L\left(\dot{m} / r_{b}^{2}\right) \exp \left(\dot{m}-\dot{m} / r_{b}\right)=\left(2 \Lambda_{o} \exp \Phi_{m}\right)^{1 / 2}
$$

and

$$
L \exp \dot{m}=\left(T_{b}-T_{\infty}\right)\left\{1-\exp \left(-\dot{m} / r_{b}\right)\right\}^{-1}
$$

This last relation states that in the outer frozen-flow regions the temperature, in the first approximation, decreases at the same rate on both sides of the thin flame.

Matching terms of order $\epsilon$, yields

$$
\begin{aligned}
& \Phi_{m}+\ln 4-\left(2 \Lambda_{o} \exp \Phi_{m}\right)^{1 / 2} \zeta_{m}=0 \\
& \Phi_{m}+\ln 4+\left(2 \Lambda_{o} \exp \Phi_{m}\right)^{1 / 2} \zeta_{m}=B_{1} .
\end{aligned}
$$

We need an additional relation for the complete determination of the unknowns $\Lambda_{o}, T_{b}, \Phi_{m}, \zeta_{m}$ and $B_{1}$. This relation may be obtained from the matching conditions between the outer expansions and the three-term expansion of the inner solution. We then obtain

$$
B_{1}=\left\{\exp \left(\dot{m} / r_{b}\right)-1\right\}\left\{2\left(T_{\infty}-T_{e}\right) Y_{b}^{-1} \exp \left(-\dot{m} / r_{b}\right)-4\left(1-2 r_{b} / \dot{m}\right)\right\}
$$

To show this we first write the differential equation for the third term of the inner expansion

$$
\begin{aligned}
\mathrm{d}^{2} \phi_{2} / \mathrm{d} \zeta^{2}= & \left(\dot{m} / r_{b}{ }^{2}-2 / r_{b}\right) \mathrm{d} \phi_{1} / \mathrm{d} \zeta-\Lambda_{o} \exp \phi_{1}\left\{\phi_{2}+b_{1}-\phi_{1}{ }^{2} Y_{b}{ }^{-1}-\phi_{1}{ }^{2} T_{b}{ }^{-1}\right. \\
& \left.+\zeta\left(T_{\infty}-T_{e}\right)\left(\dot{m} / Y_{b} r_{b}{ }^{2}\right) \exp \left(-\dot{m} / r_{b}\right)\right\}
\end{aligned}
$$

A first integration of (65) yields the equation 


$$
\begin{aligned}
\frac{\mathrm{d} \phi_{2}}{\mathrm{~d} \zeta} \frac{\mathrm{d} \phi_{1}}{\mathrm{~d} \zeta}= & -\Lambda_{o} \exp \phi_{1}\left\{\phi_{2}+b_{1}-\left(\phi_{1}-1\right) Y_{b}^{-1}-\left(\phi_{1}{ }^{2}-2 \phi_{1}+2\right) T_{b}^{-1}\right\} \\
& +\left(\dot{m} r_{b}^{-2}-2 r_{b}^{-1}\right)\left(2 \Lambda_{o} e^{\phi_{m}}+2 \frac{\mathrm{d} \phi_{1}}{\mathrm{~d} \zeta}+c\right) \\
& -\left(T_{\infty}-T_{e}\right) \frac{\dot{m}}{r_{b}^{2} Y_{b}} e^{-\dot{m} r_{b}}\left\{\Lambda_{o} \zeta e^{\phi_{1}}+\frac{\mathrm{d} \phi_{1}}{\mathrm{~d} \zeta}\right\} .
\end{aligned}
$$

The constant $c$ and the relation (64) can now be obtained from the matching conditions with the outer solutions. Thus

$$
c=\left(2 \Lambda_{o} \exp \phi_{m}\right)^{1 / 2}\left\{\left(T_{\infty}-T_{e}\right) Y_{b}^{-1}\left(1-2 r_{b} / \dot{m}\right) \exp \left(-\dot{m} / r_{b}\right)-2\right\}
$$

\section{Discussion and generalization}

We shall not undertake here a complete parametric study of droplet decomposition based on the simple results that have been obtained. The particular examples offered in Fig. 4 should be considered as illustrative.

It is clear from Fig. 4 that a regime, intermediate between the partial and complete decomposition regimes exists which would provide a smooth transition between those regimes. We have not carried out the analysis of this regime here, because of its little practical interest, as it corresponds to the unstable branch of the $\dot{m}(D)$ curve. Also, the only usefulness of the analysis of the partial decomposition regime, aside from its academic interest, lies in its ability to match with the ignition regime for $\alpha<-1$.

The results obtained in the analysis of the complete decomposition regime provide a general description of the stable modes of droplet decomposition, including the ignition conditions for small negative values of $\alpha$.

The decomposition rate, and flame position were obtained, eqns (35) and (37) in terms of the apparent flame temperature $T_{p}$. The Damköhler number is given explicitly in terms of $T_{p}$ by eqns (48) and (45). The value of $n$ in eqn (48) may be written as $n=1.344$ for the calculation of the droplet vaporization rate and extinction conditions, with very little loss of accuracy, when compared with the more accurate relation (45).

The Damköhler number for extinction can be obtained by differentiation of eqn (48) and use of the condition $\mathrm{d} D_{1}=0$ when $\mathrm{d} T_{p} \neq 0$. If the term $2 \mathrm{~d} r_{p} / r_{p}$ is neglected, compared with $T_{a} T_{p}{ }^{-2} \mathrm{~d} T_{p}$ and $n$ is considered constant $n=1.344$, we obtain the following relation

$$
\left(T_{e}-T_{p}\right) \ln \left\{\left(T_{e}-T_{\infty}\right) /\left(T_{e}-T_{p}\right)\right\}=\left(T_{a} / T_{p}+4-1.344 T_{p}\right)^{-1}
$$

giving the value of $T_{p}$ at extinction in terms of $T_{e}, T_{\infty}$, and $T_{a}$. This relation can be used together with eqns (36), (37), and (48) to calculate the Damköhler number of extinction. Notice that these relations provide an explicit parametric representation of $D_{\text {ext }}$ in terms of $T_{a}$ or $T_{\infty}$, for a given value of $T_{e}$, with $T_{p} / T_{e}$ as the parametric variable. 
It is easy to show that the extinction conditions are given by the relations

$$
\ln \left(2 D_{\mathrm{ext}} T_{e}^{4} / T_{a}^{2}\right)-T_{a} / T_{e}=\tau_{a}(1-\tau) / \tau-4 \ln \tau+1.244 T_{e}(1-\tau)+2 \ln \left(\ln \frac{1-\tau_{\infty}}{1-\tau}\right)
$$

and

$$
(1-\tau) \ln \frac{1-\tau_{\infty}}{1-\tau}=T_{e}^{-1}\left(\tau_{a} / \tau+4-1.344 T_{e}\right)^{-1}
$$

where $\tau=T_{\mathrm{p}} / T_{e}, \tau_{\infty}=T_{\infty} / T_{e}$, and $\tau_{a}=T_{a} / T_{e}$.

For given values of $T_{a}$ and $T_{e}$, eqn (70) shows that there is a maximum value of $T_{\infty}$ if we want a Damköhler number of extinctium to exist. For lower values of $T_{\infty}$ the vaporization rate increases monotonically with increasing Damköhler number. It is easy to show that for sufficiently large activation energies, a clear-cut Damköhler number of extinction will exist only if

$$
T_{e}-T_{\infty}>\left(T_{a} / T_{\infty}+4\right)^{-1} .
$$

The generalization of the asymptotic analysis carried out here to cover cases with variable transport properties and nonunity Lewis number is simple for the premixed flame regime. In this case, the conservation equations are easily integrated for variable properties, outside the reaction zone; in the thin reaction zone, in first approximation, the convective terms do not enter and the transport coefficients are constant, so that the conservation equations may be combined to yield eqn (43) as describing the reaction zone structure.

In the ignition regime there is an inner transport region, where the conservation equations may be integrated for variable properties without difficulty, and an outer reaction region where, because the convective terms do not enter in first approximation and the transport coefficients may be approximated by their values at infinity, the conservation equations may again be combined to give eqn (26) as describing the reaction zone structure.

The generalization of this asymptotic procedure for the analysis of other exothermic reactions, in more complex fluid flows with diffusion, would involve the solution in the complete decomposition regime, for example, of the frozen flow and equilibrium flow equations, and their matching to the solution of eqn (43), which would describe the structure of the reaction zone. These matching conditions would then determine the flame temperature, or the temperature of transition between the frozen flow and equilibrium flow solutions.

The multiplicity of the solutions which we obtain, is related to the quasi-steady assumption. It is clear that in an unsteady treatment of the droplet decomposition problem the solution would be uniquely related to the initial conditions. After a transient period, that may be short compared with the lifetime of the droplet, the solution would tend toward the solution corresponding to one of the stable quasi-steady regimes. 
Acknowledgment-This research has been sponsored in part by the Air Force Office of Scientific Research, through the European Office of Aerospace Research, OAR, United States Air Force, under Grants AFOSR No. 72-2254 and 73-2535A.

\section{References}

Adler, J. and Spalding, D. B. (1961) One-dimensional laminar flame propagation with an enthalpy gradient, Proc. Roy Soc. A261, 53-78.

Bush, W. B. and Fendell, F. E. (1970) Asymptotic Analysis of Laminar Flame Propagation for General Lewis Numbers, Combustion Science and Technology 1, 421.

Fendell, F. E. (1965) Finite Rate Burning of a Monopropellant Droplet in a Stagnant Atmospheric, Astronautica Acta 11, 418.

Fendell, F. E. (1969) Near-Equilibrium Decompositional Burning of Monopropellant Droplets in a Stagnant Atmosphere, Int. J. Heat Mass Transfer 12, 223.

Fendell, F. E. (1972) Asymptotic Analysis of Premixed Burning with Large Activation Energies, $J$. Fluid Mech. 56, 81.

Jain, V. K. (1963) The Theory of Burning of Monopropellant Droplets in an Atmosphere of Inerts, Combustion and Flame 7, 17-27.

Jain, V. K. and Kumar, R. N. (1969) Theory of Laminar Flame Propagation with Non-normal Diffusion, Combustion and Flame 13, 285-294.

Jain, V. K. and Ramani, N. (1969) The Theory of Burning of a Monopropellant Droplet-Variable Properties, Combustion Science and Technology 1, 1-12.

Liñán, A. (1974) The Asymptotic Structure of Counterflow Diffusion Flames for Large Activation Energies, Acta Astronautica 1(7/8), 1007-1039.

Lorell, J. and Wise, H. (1955) Steady-state Burning of a Liquid Droplet-I: Monopropellant Flame, J. Chem. Phys. 23, 1928.

Rosner, D. E. (1971) Liquid Droplet Vaporization and Combustion: In D. T. Harrje and F. H. Reardon (Eds.), ICRPG Reference Book on Combustion Instability in Liquid Rockets, Section 2.4, Princeton University.

Spalding, D. B. and Jain, V. K. (1959) Theory of Burning of Monopropellant Droplets, ARC Technical Report, C.P. No. 447.

Tarifa, C. S., del Notario, P. P. and Garcia Moreno, F. (1962) Combustion of liquid Monopropellants and Bipropellants in Droplets: In Eighth Symposium (International) on Combustion, pp. 1035-1056, Williams and Wilkins, Baltimore.

Williams, F. A. (1959) Theory of the Burning of Monopropellant Droplets, Combustion and Flame 3, 529.

Williams, F. A. (1965) Combustion Theory, Addison-Wesley, Palo Alto, Calif. 